\title{
BOUNDS FOR THE SEPARATION OF REAL ZEROS OF POLYNOMIALS
}

\author{
PETER WALKER
}

(Received 29 October, 1992)

Communicated by P. C. Fenton

\begin{abstract}
We measure the separation of the zeros of the polynomial $f(x)=\prod_{1}^{n}\left(x-a_{i}\right), a_{1}<a_{2}<\cdots<a_{n}$ by $\delta(f)=\min _{i}\left(a_{i+1}-a_{i}\right)$. We establish a bound for the amount by which the ratio $\delta\left(f^{\prime}-k f\right) / \delta(f)$ exceeds 1 .
\end{abstract}

1991 Mathematics subject classification (Amer. Math. Soc.): $26 \mathrm{C} 10$.

\section{Introduction}

The problem of the location of the zeros of a polynomial of arbitrary degree has a long history in mathematics. The number of zeros on the real axis may be investigated by Descartes' rule of signs [3, Part $5, \mathrm{Ch} .1]$, or in greater detail by means of Sturm sequences [1, Ch 6.3]. In the case of complex zeros, methods for the location of zeros in specified regions of the plane are studied in [2]. Numerical aspects of the problem can be found in [1, Ch 6].

In this paper we propose to study the separation $\left(a_{i}-a_{j}\right)$ of the zeros of a polynomial $f(x)=\prod_{1}^{n}\left(x-a_{i}\right)$. We shall measure the separation both by the minimum separation $\delta(f)=\min _{i \neq j}\left|a_{j}-a_{i}\right|$ and by the maximum or total separation $\Delta(f)=\max _{i, j} \mid a_{j}-$ $a_{i} \mid$. The quantity $\delta(f)$ gives us a measure of the closeness of the zeros so that we can detect when they are near to coincidence.

In the case when the zeros are real and distinct, we showed in [4] that $\delta(f)$ is strictly increased by the differential operator $D-k I$, that is, that $\delta\left(f^{\prime}-k f\right)>\delta(f)$, and we shall show in Section 3 of this paper that the same is true for $\Delta(f)$. In Section 2 we sharpen the result for $\delta(f)$ to give an explicit lower bound for the ratio $\delta\left(f^{\prime}-k f\right) / \delta(f)$; in particular, there is a constant $c_{n}>1$ such that $\delta\left(f^{\prime}\right) \geq c_{n} \delta(f)$.

(C) 1995 Australian Mathematical Society 0263-6115/95 \$A2.00+0.00 
Much more remains to be done in respect of improved constants, extension of the results to the complex plane and exploitation of possible symmetries among the zeros.

It is a pleasure to acknowledge the contributions of Professor R. E. Scraton for Theorem 2(i) and for extensive numerical testing.

\section{Minimum Separation}

We suppose throughout that $f(x)=\prod_{1}^{n}\left(x-a_{i}\right)$ is a polynomial of degree $n$ with distinct real zeros which we shall assume to be in increasing order: $a_{1}<a_{2}<\cdots<$ $a_{n}$. The principal result of this section is as follows:

THEOREM 1. For all $k \in \mathbb{R}$ and all polynomials $f$ of degree $n \geq 2,(n \geq 3$ if $k=0)$

$$
\delta\left(f^{\prime}-k f\right) / \delta(f)>1+\frac{4 n}{(n+2)^{2}} \cdot \frac{1}{\pi^{2}+(|k| \delta(f)+\rho+2 / n)^{2}},
$$

where $\rho=\log (4(\pi+1) /(\pi-1))=2.05 \ldots$

From Rolle's theorem the $n-1$ zeros of $f^{\prime}$ lie one in each interval $\left(a_{i}, a_{i+1}\right)$; more generally it is easy to see by considering the graph of $f^{\prime} / f$ that for given real $k$, the polynomial $f^{\prime}-k f$ has one zero, which we shall denote by $b_{i}$, in each interval $\left(a_{i}, a_{i+1}\right)$. In addition if $k>0$ there is one further zero $b_{n}>a_{n}$, while if $k<0$ there is one further zero $b_{0}<a_{1}$.

We shall say that the separation of the points $a_{i}$ is almost equal when either $a_{1}, \ldots, a_{n-1}$ are equally separated and $a_{n}\left(\geq a_{n-1}\right)$ is otherwise unrestricted, or similarly when $a_{2}, \ldots, a_{n}$ are equally separated and $a_{1}\left(\leq a_{2}\right)$ is unrestricted. It may happen that when the points $a_{i}$ vary, some of them tend to $\pm \infty$, in which case the corresponding terms are omitted from the summations.

Our plan is as follows. Firstly, we fix $j$ and find the configuration of $a_{i}$ 's which minimises $r_{j}=b_{j+1}-b_{j}$; this is done in Lemma 1 . In this configuration we estimate the position of $b_{j}$ and find lower estimates for $r_{j}$, which are stated in Lemma 2. Finally, in the proof of Theorem 1 we minimise this lower estimate over $j$.

LEMMA 1. For given $j$ and arbitrary $a_{i}$, subject to a given minimum spacing $\delta(f)$, the minimum value of $r_{j}=b_{j+1}-b_{j}$ is attained when all $a_{i}$ not at infinity are equally or almost equally spaced.

PROOF OF LEMMA 1. For given $j$ the function $r_{j}$ depends continuously on the values of $a_{i}$; this includes the possibility that some $a_{i}$ may tend to $\pm \infty$, as already noted. Since $\delta(f)$ is fixed and the results are unaffected by translation, we could assume for instance that some $a_{i}=0$, and $a_{i+1}=\delta(f)$, and so all the $a_{i}$ cannot go to $\pm \infty$. 
Hence for given $j$ we can assume that $a_{1}, a_{2}, \ldots, a_{n}$ assume the configuration which minimises $r_{j}$, and we have to show that in this configuration all $a_{i}$ not at infinity are equally or almost equally spaced.

It follows from the equation $\sum_{i=1}^{n} 1 /\left(b_{j}-a_{i}\right)=k$ that for fixed $k \in \mathbb{R}$

$$
\frac{\partial b_{j}}{\partial a_{i}}=\frac{1}{\left(b_{j}-a_{i}\right)^{2} S_{j}} \quad \text { where } \quad S_{j}=\sum_{m=1}^{n} 1 /\left(b_{j}-a_{m}\right)^{2} .
$$

Note that $0<\partial b_{j} / \partial a_{i}<1$ for all $i, j$. Hence

$$
\frac{\partial r_{j}}{\partial a_{i}}=\frac{1}{\left(b_{j+1}-a_{i}\right)^{2} S_{j+1}}-\frac{1}{\left(b_{j}-a_{i}\right)^{2} S_{j}},
$$

and this is $>0$ (respectively $=0,<0$ ) accordingly as

$$
\left(\frac{b_{j}-a_{i}}{b_{j+1}-a_{i}}\right)^{2}>(\text { or }=\text {, or }<) \frac{S_{j+1}}{S_{j}} .
$$

But the ratio $S_{j+1} / S_{j}$ is independent of $i$, while the left side, as a function of $i$, is strictly decreasing and $<1$ for $1 \leq i \leq j$, and strictly decreasing and $>1$ for $j+2 \leq i \leq n$. Hence for a given $j$ we must have one of the following three cases for the sequence of signs of $\partial r_{j} / \partial a_{i}, 1 \leq i \leq n$ :

(i) if $S_{j+1} / S_{j}>1$, the sequence of signs has the form $--\cdots-($ ?) $(++$ $\cdots+)(0)(--\cdots-)$ where the (?) is in the $(j+1)$ st position, and either the (0) or the blocks of + or - terms may be absent on the right,

(ii) if $S_{j+1} / S_{j}=1$, the sequence is $-\cdots-(?)++\cdots+$, with (?) in the $(j+1)$ st position,

(iii) if $S_{j+1} / S_{j}<1$, the sequence is $(++\cdots+)(0)(--\cdots-)($ ? $)++\cdots+$, similarly.

Notice also that if instead of varying a single $a_{i}$, we move simultaneously a block of consecutive $a_{i}$, all having the same sign for $\partial r_{j} / \partial a_{i}$, then the effect on $r_{j}$ of either an increase or a decrease will be the same as for a move in the individual terms; this also holds if one of the signs is zero.

We now consider case (i) in detail and suppose initially that $j \geq 1$ so that there is at least one $\partial r_{j} / \partial a_{i}$ with $i<j+1$, and all these derivatives must be $<0$. Then all intervals $\left(a_{i}, a_{i+1}\right)$ with $i \leq j$ must be of length $\delta(f)$, since otherwise $r_{j}$ could be decreased by a simultaneous increase in $a_{1}, \ldots, a_{i}$. Now look at the signs to the right of $j+1$. These cannot end with a - since if they did, then $r_{j}$ could be decreased by an increase in $a_{n}\left(\delta(f)\right.$ being already attained to the left of $\left.a_{j+1}\right)$. Hence the sign sequence must be $-\cdots-(?)(++\cdots+)(0)$. If there is zero at $a_{n}$ we have no control on the length of $\left(a_{n-1}, a_{n}\right)$. Apart from this, all intervals $\left(a_{i}, a_{i+1}\right)$ with a + at 
$a_{i+1}$ must be of length $\delta(f)$ since otherwise $r_{j}$ could be decreased by a simultaneous decrease in $a_{i+1}, \ldots, a_{n}$. This proves equal or almost equal spacing in this case.

In the case $j=0$ the sign sequence is $(?)(++\cdots+)(0)(--\cdots-)$. Now if the last sign is -, then the last interval $\left(a_{n-1}, a_{n}\right)$ must have length $\delta(f)$, otherwise $r_{j}$ is decreased by an increase in $a_{n}$. Then there can be no preceeding 0 or - signs, since if there were, then $r_{j}$ could be decreased by a simultaneous increase of all terms with a 0 or - sign. Hence the only remaining possibilities are either (?) $++\cdots+(0)$, which we showed above leads to equal or almost equal spacing, or (?) $++\cdots+-$, which we shall show cannot occur. We can assume that $n \geq 4$ since equal or almost equal spacing is automatic if $n=2$ or 3 . Then $\partial r_{j} / \partial a_{2}>0$, and we know that $\delta(f)$ is already attained on $\left(a_{n-1}, a_{n}\right)$ where $n-1 \geq 3$. Hence if $a_{2}-a_{1}>\delta(f)$ then a decrease in $a_{2}$ (only!) will decrease $r_{j}$. Hence $\delta(f)$ is attained also on $\left(a_{1}, a_{2}\right)$ and so as above there can be no - sign at $a_{n}$.

Cases (ii) and (iii) are treated similarly, and Lemma 1 is proved.

NOTE. Numerical examples show that at least three possibilities can occur. For instance if $n=4$ and $a_{i}=i, 1 \leq i \leq 3, a_{4}=4+t$, and $k=-1$, then $r_{0}$ is a decreasing, and $r_{2}$ is an increasing function of $t$, while $r_{1}$ is firstly increasing and then decreasing with a maximum near $t=6$.

From now on we shall study the case of equal separation in detail. Almost equal separation will not be considered again until the latter part of the proof of Theorem 1.

Our next objective is to estimate the positions of the points $b_{j}$. To find these we write $b_{j}=a_{j}+t_{j}$ for all $j \geq 1$; we can simplify by putting $\delta(f)=1$ and $a_{i}=i$ for $1 \leq i \leq n$. Then we have $b_{j}=j+t_{j}$ and we can also put $t_{0}=b_{0}$. Then we have the following estimates for $t_{j}$ and $t_{j+1}-t_{j}$ in the normalised case.

LEMMA 2. Let $E(t)=\pi \cot \pi t$ and let $E^{-1}$ be the branch of the inverse with values in the interval $(0,1): E^{-1}(y)=1 / 2-\tan ^{-1}(y / \pi) / \pi$. Let $h_{n}$ denote the harmonic number $1+1 / 2+\cdots+1 / n$. Then we have the following estimates for $t_{j}$ :

(i)(a) $t_{j}>E^{-1}\left(k+h_{n-j}-h_{j-1}\right)=p_{j}$ say, for $1 \leq j \leq n$,

(b) $t_{j}<E^{-1}\left(k+h_{n-j-1}-h_{j}\right)=q_{j}\left(=p_{j+1}\right)$ say, for $0 \leq j \leq n-1$.

We have $t_{j+1}-t_{j}>n /\left[(n-j)(j+1)\left(\pi^{2}+k^{2}\right)\right]$ where

$$
k^{\prime}= \begin{cases}k+h_{n-2}-1, & \text { for } j=0 \\ \max \left(\left|k+h_{n-j}-h_{j-1}\right|,\left|k+h_{n-j-2}-h_{j+1}\right|\right), & \text { for } 1 \leq j \leq n-2 \\ k+1-h_{n-2}, & \text { for } j=n-1 .\end{cases}
$$

Note that (ii) gives us a proof that $t_{j+1}>t_{j}$, which is the special case of $\delta\left(f^{\prime}-k f\right)>$ $\delta(f)$ for equal spacing. 
ProOF OF LemMa 2. (i) We know that $t_{j}$ is defined as a solution of the equation $\psi_{j}(t)=\sum_{1-j}^{n-j} 1 /(t-r)=k$. Write $E(t)=\pi \cot \pi t=\psi_{j}(t)+\Psi_{j}(t)$. For sufficiently large $N, \Psi_{j}(t)$ can be written

$$
\Psi_{j}(t)=\sum_{r=-N}^{-j} \frac{1}{t-r}+\sum_{r=n-j+1}^{N} \frac{1}{t-r}+\sum_{r=N+1}^{\infty}\left(\frac{1}{t-r}+\frac{1}{t+r}\right),
$$

and from the periodicity of $E(t)$ it follows that

$$
\Psi_{j}(t+1)=\Psi_{j}(t)+\frac{1}{t+j-n}-\frac{1}{t+j} .
$$

In particular $\Psi_{j}(0)=h_{n-j}-h_{j-1}$ and $\Psi_{j}(1)=h_{n-j-1}-h_{j}$ whenever these are defined.

But $E\left(t_{j}\right)=k+\Psi_{j}\left(t_{j}\right)$ and $\Psi_{j}$ is decreasing on its intervals of continuity. Hence since $t_{j}>0$ for $1 \leq j \leq n$ we have $E\left(t_{j}\right)<k+\Psi_{j}(0)$, or $t_{j}>E^{-1}\left(k+h_{n-j}-h_{j-1}\right)$. Similarly for $0 \leq j \leq n-1$ we have $t_{j}<1$ and so $k+\Psi_{j}(1)<E\left(t_{j}\right)$ and $t_{j}<E^{-1}\left(k+h_{n-j-1}-h_{j}\right)$.

(ii) Note that

$$
\sum_{i=1}^{n} \frac{1}{b_{j}-a_{i}}=\sum_{i=1}^{n} \frac{1}{j+t_{j}-i}=k=\sum_{i=1}^{n} \frac{1}{b_{j+1}-a_{i}}=\sum_{i=1}^{n} \frac{1}{j+1+t_{j+1}-i} .
$$

Let $\phi_{j}(t)=\sum_{i=1}^{n-1} 1 /(j+t-i)$, so that $\phi_{j}$ is decreasing on each interval of continuity, and the above can be written

$$
\begin{aligned}
\frac{1}{j-n+t_{j}}+\phi_{j}\left(t_{j}\right) & =k=\frac{1}{j+t_{j+1}}+\phi_{j}\left(t_{j+1}\right), \quad \text { or } \\
\phi_{j}\left(t_{j+1}\right)-\phi_{j}\left(t_{j}\right) & =-\frac{n+t_{j+1}-t_{j}}{\left(n-j-t_{j}\right)\left(j+t_{j+1}\right)},
\end{aligned}
$$

which is negative, since $t_{j+1}>t_{j}$ as already noted. In particular we have $\mid \phi_{j}\left(t_{j+1}\right)-$ $\phi_{j}\left(t_{j}\right) \mid>n /[(n-j)(j+1)]$ for $0 \leq j \leq n-1$.

But from the mean value theorem we have $t_{j+1}-t_{j}=\left(\phi_{j}\left(t_{j+1}\right)-\phi_{j}\left(t_{j}\right)\right) / \phi_{j}^{\prime}(\theta)$ for some $\theta \in\left(t_{j}, t_{j+1}\right)$ and

$$
\left|\phi_{j}^{\prime}(\theta)\right|=\sum_{i=1}^{n-1} \frac{1}{(j+\theta-i)^{2}}<\frac{\pi^{2}}{\sin ^{2} \pi \theta},
$$

so that for $1 \leq j \leq n-2$,

$$
\begin{aligned}
t_{j+1}-t_{j} & >\frac{n}{\pi^{2}(n-j)(j+1)} \inf \left\{\sin ^{2} \pi \theta: t_{j} \leq \theta \leq t_{j+1}\right\} \\
& =\frac{n}{\pi^{2}(n-j)(j+1)} \min \left\{\sin ^{2} \pi t_{j}, \sin ^{2} \pi t_{j+1}\right\} \\
& >\frac{n}{\pi^{2}(n-j)(j+1)} \min \left\{\sin ^{2} \pi p_{j}, \sin ^{2} \pi q_{j+1}\right\} .
\end{aligned}
$$


Since $E\left(p_{j}\right)=\pi \cot \pi p_{j}=k+h_{n-j}-h_{j-1}$ it follows that

$$
\begin{aligned}
\sin ^{2} \pi p_{j} & =\frac{\pi^{2}}{\pi^{2}+\left(k+h_{n-j}-h_{j-1}\right)^{2}}, \quad \text { and similarly } \\
\sin ^{2} \pi q_{j+1} & =\frac{\pi^{2}}{\pi^{2}+\left(k+h_{n-j-2}-h_{j+1}\right)^{2}} .
\end{aligned}
$$

Hence

$$
t_{j+1}-t_{j}>\frac{n}{(n-j)(j+1)} \frac{1}{\pi^{2}+k^{\prime 2}}
$$

where $k^{\prime}=\max \left(\left|k+h_{n-j}-h_{j-1}\right|,\left|k+h_{n-j-2}-h_{j+1}\right|\right)$, as required, if $1 \leq j \leq$ $n-2$.

When $j=n-1$ we have $\left|\phi_{j}^{\prime}(\theta)\right|=\sum_{i=0}^{n-2} 1 /(\theta+i)^{2}$ which is positive and decreasing on $(0, \infty)$. Hence since $t_{n}>\theta>t_{n-1}>p_{n-1}>0$ we have

$$
\left|\phi_{j}^{\prime}(\theta)\right|<\left|\phi_{j}^{\prime}\left(t_{n-1}\right)\right|<\left|\phi_{j}^{\prime}\left(p_{n-1}\right)\right|<\sin ^{2}\left(\pi p_{n-1}\right) / \pi^{2}=1 /\left(\pi^{2}+\left(k+1-h_{n-2}\right)^{2}\right)
$$

as required. The case $j=0$ is similar, and the proof of Lemma 2 is complete.

Before we can go on to the proof of Theorem 1, we need the following technicality.

LEMMA 3. Let $G(t)=t(1-t)\left[\pi^{2}+\{k+\log ((1-t) / t)\}^{2}\right]$, for $t \in(0,1)$, and $G(0)=G(1)=0$.

Then $G(t) \leq\left(\pi^{2}+(|k|+c)^{2}\right) / 4$ where $c=\log ((\pi+1) /(\pi-1))$.

ProOf OF LeMMA 3. Suppose that $k \geq 0$ without loss of generality. Then $G(t)$ is continuous and non-negative on $[0,1]$ and $G(t) \geq G(1-t)$ for $0 \leq t \leq 1 / 2$, so that in looking for a maximum we need consider only the interval $[0,1 / 2]$. We have $G^{\prime}(t)=0$ when

$$
0=\frac{1}{t}-\frac{1}{1-t}+\frac{2 \theta}{\pi^{2}+\theta^{2}}\left(-\frac{1}{1-t}-\frac{1}{t}\right)
$$

where $\theta=k+\log ((1-t) / t)$, or $1-2 t=2 \theta /\left(\pi^{2}+\theta^{2}\right)$. But $2 \theta /\left(\pi^{2}+\theta^{2}\right) \leq 1 / \pi$, so we must have $1 / 2 \geq t \geq t_{0}=(1-1 / \pi) / 2$.

Hence we can get an upper bound for $G(t)$ by maximising $t(1-t)$ at $t=1 / 2$, and by maximising $\pi^{2}+\theta^{2}$ at $t=t_{0}$, which gives $G(t) \leq\left(\pi^{2}+(k+\log ((\pi+1) /(\pi-1)))^{2}\right) / 4$ as required.

PROOF OF THEOREM 1. The proof is in two parts. In the first we assume equal separation and use Lemmas 2 and 3 to obtain the result. In the second we consider what modifications are needed to deal with the case of almost equal separation. 
We can suppose that $k \geq 0$ since the result is the same for $k$ and $-k$. We have to minimise over $j$ the various estimates for $t_{j+1}-t_{j}$ which appear in Lemma 2. More precisely we have to find upper bounds for

$f_{1}(j)=(n-j)(j+1)\left(\pi^{2}+\left(k+h_{n-j-2}-h_{j+1}\right)^{2}\right) \quad$ for $0 \leq j \leq n-2, \quad$ and $f_{2}(j)=(n-j)(j+1)\left(\pi^{2}+\left(k+h_{n-j}-h_{j-1}\right)^{2}\right) \quad$ for $1 \leq j \leq n-1$.

We consider $f_{1}$ firstly and deduce the results for $f_{2}$. We use repeatedly the elementary inequalities

$$
0 \leq \log \frac{m+1}{n+1} \leq h_{m}-h_{n} \leq \log \frac{m+\frac{1}{2}}{n+\frac{1}{2}}
$$

which are valid for all integers $m \geq n \geq 0$.

In dealing with $f_{1}$ we first consider the values of $j$ for which $k+h_{n-j-2}-h_{j+1} \geq 0$, and we bound this above by

$$
k+\log \frac{n-j-\frac{3}{2}}{j+\frac{3}{2}} \quad \text { or } \quad k+\log \frac{n-j-1}{j+2}
$$

accordingly as $j+1 \leq n-j-2$ or not. But

$$
\begin{aligned}
(n- & j)(j+1)\left(\pi^{2}+\left(k+\log \frac{n-j-\frac{3}{2}}{j+\frac{3}{2}}\right)^{2}\right) \\
& \leq(n-j)\left(j+\frac{3}{2}\right)\left(\pi^{2}+\left(k+\log \frac{n-j}{j+\frac{3}{2}}\right)^{2}\right) \\
& \leq\left(n+\frac{3}{2}\right)^{2}\left(\pi^{2}+(k+c)^{2}\right) / 4,
\end{aligned}
$$

by Lemma 3 with $t=(j+3 / 2) /(n+3 / 2)$; similarly

$$
(n-j)(j+1)\left(\pi^{2}+\left(k+\log \frac{n-j-1}{j+2}\right)^{2}\right) \leq(n+2)^{2}\left(\pi^{2}+(k+c)^{2}\right) / 4
$$

by Lemma 3 with $t=(j+2) /(n+2)$.

For values of $j$ with $k+h_{n-j-2}-h_{j+1}<0$ (which certainly requires $j+1>n-j-2$ ) we have $\left|k+h_{n-j-2}-h_{j+1}\right|<k+h_{j+1}-h_{n-j-2}<k+\log \left[\left(j+\frac{3}{2}\right) /\left(n-j-\frac{3}{2}\right)\right]$, and so

$$
f_{1}(j)<(n-j)(j+1)\left(\pi^{2}+\left(k+\log \frac{j+\frac{3}{2}}{n-j-\frac{3}{2}}\right)^{2}\right)
$$




$$
\begin{aligned}
& =(n-j)(j+1)\left(\pi^{2}+\left(k+\log \frac{j+\frac{3}{2}}{n-j}+\log \frac{n-j}{n-j-\frac{3}{2}}\right)^{2}\right) \\
& \leq(n-j)(j+1)\left(\pi^{2}+\left(k+\log 4+\log \frac{j+\frac{3}{2}}{n-j}\right)^{2}\right) \\
& <\left(n+\frac{3}{2}\right)^{2}\left(\pi^{2}+(k+\log 4+c)^{2}\right) / 4,
\end{aligned}
$$

using Lemma 3 with $k+\log 4$ for $k$ and $t=(n-j) /(n+3 / 2)$. The consideration of $f_{2}$ is reduced to that of $f_{1}$ by putting $n-j-1$ for $j$ and $-k$ for $k$. This completes the proof of Theorem 1 in the case of equal separation when $d=\delta(f)=1$, giving a slightly stronger result in which the $2 / n$ term is absent in the denominator. For the general case in which $d>0$ is unrestricted, we rescale, replacing $\delta\left(f^{\prime}-k f\right)$ by $\delta\left(f^{\prime}-k f\right) / d$ and $k$ by $k d$, and the result follows for this case.

To show how the proof must be modified in the case of almost equal separation we use the following lemma which refers back to the initial situation in which the points $a_{i}$ are unrestricted.

LEMMA 4. For $1 \leq j \leq n$ write $b_{j}=a_{j}+t_{j}$ and $b_{j-1}=a_{j}-u_{j}$, so that $t_{1}, \ldots, t_{n-1}$ and $u_{1}, \ldots, u_{n-1}$ are defined for all $k$, while $t_{n}$ exists only for $k>0$, and $u_{1}$ only for $k<0$. Let $\sigma_{j}=\sum_{k \neq j} 1 /\left(a_{j}-a_{k}\right), \sigma_{j}^{*}=\sum_{k \neq j} 1 /(j-k) d=\left(h_{j-1}-h_{n-j}\right) / d$ where $d=\delta(f)$, and let $F_{j}(t)=\sum_{i=1}^{n} 1 /(t+(j-i) d)$ for $0<t<d$, so that $F_{j}$ is a continuous decreasing function from $(0, d)$ onto $\mathbb{R}$.

Then (i) $t_{j} \geq F_{j}^{-1}\left(k+\sigma_{j}^{*}-\sigma_{j}\right)$, and (ii) $u_{j} \geq d-F_{j-1}^{-1}\left(k+\sigma_{j}^{*}-\sigma_{j}\right)$.

Note that if we add the results of (i) and (ii) and use the result that $F_{j}^{-1}>F_{j-1}^{-1}$ which follows from Lemma 2(i), we obtain $t_{j}+u_{j}>d$; that is, we have obtained a new proof of $\delta\left(f^{\prime}-k f\right)>\delta(f)$ along completely different lines from the one in [4].

PROOF OF LEMMA 4. The equation $\sum_{1}^{n} 1 /\left(x-a_{i}\right)=k$ is satisfied when $x=a_{j}+t_{j}$, so

$$
\sum_{1}^{n} 1 /\left(t_{j}+a_{j}-a_{i}\right)=k \text { and consequently }-d k / d t_{j}=\sum_{1}^{n} 1 /\left(t_{j}+a_{j}-a_{i}\right)^{2} .
$$

Hence for $0<t_{j}<d$,

$$
-\frac{d k}{d t_{j}} \leq \sum_{1}^{n} \frac{1}{\left(t_{j}+(j-i) d\right)^{2}}
$$




$$
\begin{aligned}
\frac{d}{d t_{j}}\left(\frac{1}{t_{j}}-k\right) & \leq \frac{d}{d t_{j}}\left(\sum_{i \neq j} \frac{-1}{t_{j}+(j-i) d}\right), \quad \text { which gives } \\
\frac{1}{t_{j}}-k+\sigma_{j} & \leq \sum_{i \neq j}\left(\frac{1}{(j-i) d}-\frac{1}{t_{j}+(j-i) d}\right)
\end{aligned}
$$

on integration from 0 to $t_{j}$. But the last equation gives

$$
k+\sigma_{j}^{*}-\sigma_{j} \geq \sum_{1}^{n} 1 /\left(t_{j}+(j-i) d\right)=F_{j}\left(t_{j}\right)
$$

as required for (i); the proof of (ii) is similar.

The proof of Theorem 1 can now be completed quickly. Suppose that in the case of almost equal separation we have $a_{i}=i d$ for $1 \leq i \leq n-1$ and $a_{n} \geq n d$. Then

$$
0>\sigma_{j}^{*}-\sigma_{j}=\frac{1}{(j-n) d}-\frac{1}{j d-a_{n}}>\frac{-1}{(n-j) d} .
$$

But in the proof of Lemma 3 the maxima of $f_{1}$ and $f_{2}$ are attained when $1 \leq j \leq n / 2$, and so we have $0>\sigma_{j}^{*}-\sigma_{j}>-2 / n d$. Putting this into (i) and (ii) of Lemma 4 shows that we can use the estimates for the equally spaced configuration (given by the functions $F_{j}^{-1}$ ) with a value of $k$ which is altered by at most $2 /(n d)$ and this proves the stated result in full.

Concerning the best value of the ratio in Theorem 1, we can make the following observations. For the case of equal spacing and $\delta(f)=1$, we define $c_{n}(k)=\min _{j} r_{j}=$ $\min _{j}\left(b_{j+1}-b_{j}\right)$ for any $k \in \mathbb{R}$. It follows from $\delta\left(f^{\prime}-k f\right)>\delta(f)$ that $c_{n}(k)>1$ and we conjecture that $c_{n}(k d)$ gives the best value of the ratio in Theorem 1; that is that equal separation always gives the configuration which minimises $r_{j}$.

Since each $b_{j}$ is a real-analytic function of $k, c_{n}(k)$ is piecewise analytic. Simple asymptotic calculations show both that $r_{j} \simeq 1+n /\left(k^{2} j(n-j)\right)$ for fixed $j$ as $k \rightarrow+\infty$ (and a similar result at $-\infty$ ), and that for $k=0, c_{n}(0) \simeq 1+4 /\left(\pi^{2} n\right)$ as $n \rightarrow \infty$. This shows that Theorem 1 gives at least the correct orders of magnitude.

However it is certainly not the case that the minimum of $r_{j}$ is always attained for the same value of $j$; it happens even in the smallest interesting cases $(n=4,5)$ that the location of the minimum changes from one branch to another as $k$ varies. Hence if, as we conjecture, $c_{n}(k)$ is really the best value of the ratio in Theorem 1 , then this cannot be given by a single real-analytic function at all points.

There can be no upper bound of the form $\delta\left(f^{\prime}-k f\right) \leq$ (constant) $\delta(f)$ since $\delta(f)$ may tend to 0 while $\delta\left(f^{\prime}-k f\right)$ remains bounded away from 0 . However for $k \neq 0$ we have trivially $\delta\left(f^{\prime}-k f\right) \leq \delta(f)+n /|k|$; the following argument shows 
slightly more, namely, that $\delta\left(f^{\prime}-k f\right)^{2} \leq \delta(f)^{2}+(n / k)^{2}$. A stronger result, that $\delta\left(f^{\prime}-k f\right)<\delta(f)+\mathrm{O}(\log n \log \log n /|k|)$ will appear in [5].

Suppose then that $k>0$ is given and that $j$ is chosen so that $\delta(f)=a_{j+1}-a_{j}$ : say $a_{j}=0, a_{j+1}=d=\delta(f)$. Then we have $0=a_{j}<b_{j}<d=a_{j+1}<b_{j+1}$ and $\delta\left(f^{\prime}-k f\right) \leq b_{j+1}-b_{j}$.

However when we fix the positions of $a_{j}, a_{j+1}$ while allowing $j$ and the other $a_{i}$ to vary unrestrictedly, then the maximum of $b_{j+1}$ occurs when $j+1=n$, while $a_{1}<\cdots<a_{n-1}$ are all close to zero, so that

$$
b_{j+1}<\frac{1}{2}\left\{(d+n / k)+\sqrt{(d+n / k)^{2}-4(n-1) d / k}\right\} .
$$

Similarly the minimum of $b_{j}$ is when $j=1$ and all $a_{2}<\cdots<a_{n}$ are close to $d$, so that $b_{j}>\left\{(d+n / k)-\sqrt{(d+n / k)^{2}-4 d / k}\right\} / 2$, and we deduce that

$$
\begin{aligned}
\delta\left(f^{\prime}-k f\right) & \leq \frac{1}{2}\left\{\sqrt{(d+n / k)^{2}-4(n-1) d / k}+\sqrt{(d+n / k)^{2}-4 d / k}\right\} \\
& \leq \sqrt{d^{2}+(n / k)^{2}} .
\end{aligned}
$$

Similar estimates occur in the next section of the paper when we consider the total separation $\Delta(f)$.

\section{Total Separation}

For $\Delta(f)=a_{n}-a_{1}$ we have different results for $f^{\prime}$ and for $f^{\prime}-k f,(k \neq 0)$. The restriction to polynomials having only real zeros still applies.

Notice that in this section we have to take account of repeated zeros of $f$, a possibility that obviously did not occur in Section 2 . To handle this we denote the distinct zeros of $f$ by $\alpha_{i}, 1 \leq i \leq m$, say where $\alpha_{i}$ is repeated $n_{i}$ times so that $\sum_{1}^{m} n_{i}=n$, and let $\beta_{i}$ be the zero of $f^{\prime}$ which lies in the interval $\left(\alpha_{i}, \alpha_{i+1}\right)$.

THEOREM 2. (i) For all polynomials of degree $n \geq 3$ we have

$$
\sqrt{\frac{n-2}{n}} \Delta(f) \leq \Delta\left(f^{\prime}\right) \leq \Delta(f)
$$

There is equality on the left if and only if $f(x)=x(x-1 / 2)^{n-2}(x-1)$, taking $\alpha_{1}=0$, $\alpha_{m}=1$. There is equality on the right if and only if both $a_{1}$ and $a_{n}$ are repeated zeros of $f$. 
(ii) If $k \neq 0$ we have on putting $\Delta(f)=d$ that

$$
\begin{aligned}
d=\Delta(f) & <\frac{1}{2}\left\{\sqrt{(d+n /|k|)^{2}-4 d /|k|}+\sqrt{(d+n /|k|)^{2}-4(n-1) d /|k|}\right\} \\
& <\Delta\left(f^{\prime}-k f\right)<\frac{1}{2}\left\{(d+n /|k|)+\sqrt{(d+n /|k|)^{2}-4 d /|k|}\right\} .
\end{aligned}
$$

ProOF. (i) The inequality $\Delta\left(f^{\prime}\right) \leq \Delta(f)$ is immediate from Rolle's theorem; we shall not consider it further.

The other inequality is trivially true when both $\alpha_{1}$ and $\alpha_{m}$ are repeated. If only one of them (say $\alpha_{1}$ ) is repeated then $\Delta\left(f^{\prime}\right)=\beta_{m-1}$, and as in the proof of Lemma 1 we have $\partial \beta_{m-1} / \partial \alpha_{i}>0$ for all $i, 2 \leq i \leq m-1$, and it follows that no such $\alpha_{i}$ can exist in the minimum configuration. This means that we need only consider $f(x)=x^{n-1}(x-1)$ in which case $\Delta\left(f^{\prime}\right)=(n-1) / n$ which is $\geq \sqrt{(n-2) / n}$ as required.

The more interesting case is when neither $\alpha_{1}$ nor $\alpha_{m}$ are repeated. Then $f$ has the form $f(x)=x \prod_{2}^{m-1}\left(x-\alpha_{i}\right)^{n_{i}}(x-1)$, which gives

$$
\frac{f^{\prime}(x)}{f(x)}=\frac{1}{x}+\sum_{2}^{m-1} \frac{n_{i}}{x-\alpha_{i}}+\frac{1}{x-1}
$$

which is equal to zero if $x=\beta_{j}$ and so

$$
\frac{\partial \beta_{j}}{\partial \alpha_{i}}=\frac{n_{i}}{\left(\beta_{j}-\alpha_{i}\right)^{2} S_{j}} \quad \text { with } \quad S_{j}=\frac{1}{\beta_{j}^{2}}+\sum_{2}^{m-1} \frac{n_{i}}{\left(\beta_{j}-\alpha_{i}\right)^{2}}+\frac{1}{\left(\beta_{j}-1\right)^{2}} .
$$

Consequently $\partial\left(\beta_{m-1}-\beta_{1}\right) / \partial \alpha_{i}$ is $>0$ (or $=0$ or $<0$ ) according to whether

$$
\left.\left(\frac{\beta_{1}-\alpha_{i}}{\beta_{m-1}-\alpha_{i}}\right)^{2}>\text { (or }=\text {, or }<\right) \frac{S_{m-1}}{S_{1}} .
$$

But $S_{m-1} / S_{1}$ is independent of $i$, while the left side is a strictly increasing function of $i$ for $2 \leq i \leq m-2$. It follows that in the minimum configuration at most one of these derivatives can equal zero and so $f$ must have the form $x(x-\alpha)^{n-2}(x-1)$. In this case we find that $\beta_{1,2}=\left(2 \alpha-1+n \pm \sqrt{(2 \alpha-1+n)^{2}-4 \alpha n}\right) /(2 n)$, so $\Delta\left(f^{\prime}\right)=\sqrt{(2 \alpha-1+n)^{2}-4 \alpha n} / n$ which is minimised when $\alpha=1 / 2$ and (i) is proved.

(ii) We take $k>0$ and $\alpha_{1}=0$ without loss of generality. Then $f^{\prime}-k f$ has one further zero $\beta_{m}>\alpha_{m}$, and $\Delta\left(f^{\prime}-k f\right)=\beta_{m}-\alpha_{1}$ if $\alpha_{1}$ is repeated, $\Delta\left(f^{\prime}-k f\right)=$ $\beta_{m}-\beta_{1}$ otherwise.

In the first case, since $\partial \beta_{m} / \partial \alpha_{i}>0$ for all $i$ with $1<i<m$, no such $\alpha_{i}$ can exist in either a minimum or a maximum configuration. Hence, writing $a_{n}=\alpha_{m}=\Delta(f)=d$, 
$\beta_{m}$ is minimised when $f(x)=x^{n-1}(x-d)$, and maximised when $f(x)=x^{2}(x-d)^{n-2}$, giving

$$
\begin{aligned}
\frac{1}{2}\{(d+n / k) & \left.+\sqrt{(d+n / k)^{2}-4(n-1) d / k}\right\} \leq \Delta\left(f^{\prime}-k f\right) \\
& \leq \frac{1}{2}\left\{(d+n / k)+\sqrt{(d+n / k)^{2}-8 d / k}\right\}
\end{aligned}
$$

when $\alpha_{1}$ is repeated.

The second case in which $\alpha_{1}$ is not repeated is more difficult. The proof can proceed as in (i) above to the point where there can be at most one variable point $\alpha$, $0<\alpha<d=\Delta(f)$, so that $f(x)=x(x-\alpha)^{n-r}(x-d)^{r-1}$, for some $r, 1<r \leq n$. However the determination of $\beta_{1}$ and $\beta_{3}$ requires the explicit calculation of the roots of a cubic equation, and finding the value of $\alpha$ to give a stationary value of $\beta_{3}-\beta_{1}$ turns out to be impossibly cumbersome.

We fall back on some approximations. The maximum and minimum values of $\beta_{3}$ are given respectively when $\alpha=d$ and when $\alpha$ is (arbitrarily close to) zero, with $r=2$ in the latter case. This gives the range of values

$$
\begin{aligned}
\frac{1}{2}\{(d+n / k) & \left.+\sqrt{(d+n / k)^{2}-4(n-1) d / k}\right\}<\beta_{3} \\
& \leq \frac{1}{2}\left\{(d+n / k)+\sqrt{(d+n / k)^{2}-4 d / k}\right\} .
\end{aligned}
$$

The same configurations give minimum and maximum values of $\beta_{1}$ :

$$
0<\beta_{1} \leq \frac{1}{2}\left\{(d+n / k)-\sqrt{(d+n / k)^{2}-4 d / k}\right\} .
$$

Combining these gives

$$
\begin{aligned}
\frac{1}{2}\left\{\sqrt{(d+n / k)^{2}-4 d / k}\right. & \left.+\sqrt{(d+n / k)^{2}-4(n-1) d / k}\right\}<\beta_{3}-\beta_{1}=\Delta\left(f^{\prime}-k f\right) \\
& <\frac{1}{2}\left\{(d+n / k)+\sqrt{(d+n / k)^{2}-4 d / k}\right\},
\end{aligned}
$$

and the result follows on combining (1) and (2).

NOTE. It is easy to check that the expression on the left of (2) is greater than $d$ and less than the left side of (1), so we have shown in particular that if $k \neq 0$ then $\Delta\left(f^{\prime}-k f\right)>\Delta(f)$ as claimed in the introduction. 


\section{References}

[1] P. Henrici, Applied and computational complex analysis, I (Wiley, New York, 1974).

[2] M. Marden, Geometry of polynomials (Amer. Math. Soc., Providence, 1965).

[3] G. Szegö and G. Pólya, Problems and theorems in analysis, II (Springer, New York, 1976).

[4] P. L. Walker, 'Separation of the zeros of polynomials', Amer. Math. Monthly, 100 (1993), 272-273.

[5] —_, 'Upper bounds for separation of real zeros of polynomials', Proc. Edinburgh Math. Soc., to appear.

College of Science, P.O. 36

Sultan Qaboos University

Al-Khod, 123

Muscat, Sultanate of Oman 